of heart failure was lower in this study than in previous trials of specialized management: only half of the patients were listed as NYHA class III or IV at baseline.

Half of the patients in each group were hospitalized at least once during the first year of the study. Heart failure was the reason for rehospitalization in $32 \%$ and $37 \%$ of patients in the intervention and control groups, respectively, and the time to first rehospitalization for heart failure did not differ significantly between the two groups. The rates of rehospitalization for other causes were also similar in both groups.

DeBusk et al. conclude that supplementing usual care with a nurse care management program did not reduce the rate of rehospitalization in these low-risk patients. They add that this type of management program might be beneficial in non-HMO settings.

Original article DeBusk RF et al. (2004) Care management for low-risk patients with heart failure. Ann Intern Med 141: 606-613

\section{Body-mass index in secondary prevention}

Primary prevention studies have linked BMI to the risk of cardiovascular and all-cause mortality. It is unclear, however, whether this association also applies to patients with pre-existing cardiovascular disease. Widlansky et al. have addressed this question in a large study.

As part of the Physicians' Health Study (PHS) in the US, 5,010 male physicians with a history of $\mathrm{Ml}$ or stroke returned baseline enrollment questionnaires and were included in the PHS Enrollment Cohort. Self-reported values for BMI were obtained at baseline from each participant and cause-specific deaths were identified over a mean period of 5 years. Men were stratified by smoking status and multivariate analyses accounted for other risk factors such as age, diabetes and hypertension.

Compared with the referent range of 22.0$24.9 \mathrm{~kg} / \mathrm{m}^{2}$, men with BMI values higher than $28.0 \mathrm{~kg} / \mathrm{m}^{2}$ had a similar risk of total or cardiovascular mortality, despite being overweight or obese according to World Health Organization recommendations. Interestingly, men with
BMI values lower than $22.0 \mathrm{~kg} / \mathrm{m}^{2}$ showed a 1.4-fold increase in the risk of total and cardiovascular mortality.

Widlanksy et al. conclude that elevated BMI was not strongly related to the risk of all-cause or cardiovascular mortality in this secondary prevention study. The presence of atherosclerosis and severity of underlying cardiovascular disease may attenuate any association between BMI and mortality. Since these results contrast with those from primary prevention trials, the authors suggest that further studies are warranted.

Original article Widlansky ME et al. (2004) Body mass index and total and cardiovascular mortality in men with a history of cardiovascular disease. Arch Intern Med 164: 2326-2332

\section{Lack of consensus on preoperative cardiac risk management}

Despite the publication of American College of Cardiology/American Heart Association guidelines in 2002, there is still disagreement on the most appropriate strategy for the preoperative cardiac risk management of patients undergoing noncardiac surgery. Pierpont and colleagues have recently demonstrated differences of opinion and deviations from the guidelines among a sample of US cardiologists.

The authors selected 12 cases from four distinct subsets of the ongoing Coronary Artery Revascularization Prophylaxis (CARP) trial, which was set up to assess the longterm benefit of preoperative coronary artery revascularization in patients with cardiac problems who were scheduled for vascular surgery. They then recruited 31 practicing cardiologists from three different regions, who independently reviewed the case records. The cardiologists were asked to provide recommendations on how each case should be managed; these were recorded on a scale from 1 (no revascularization prior to elective vascular surgery) to 7 (strongly recommend preoperative percutaneous intervention or bypass surgery).

Although the cardiologists' opinions agreed in some cases, there were frequent differences of opinion: recommendations from any two 